\title{
Migration of Organic Contaminants from Landfill: Minimum Thickness of Barriers
}

\author{
Bharat Jhamnani* and S.K. Singh
}

Department of Civil \& Environmental Engineering, Delhi College of Engineering, Delhi, India

\begin{abstract}
Point sources such as landfills, can release high concentrations of contaminants into the groundwater because of migration of leachate from its bottom, which is generated primarily as a result of precipitation falling on an active landfill surface, leaching out the potential organic and inorganic contaminants from landfilled waste and discharging the same to groundwater in underlying aquifer. Leachate from young landfills has high dissolved solids content as well as a high concentration of organic matter. Landfill leachate has the potential to contaminate the surrounding environment and impair groundwater use. Organic contaminants in landfill leachate originate from incoming wastes or are produced as a result of biochemical reactions taking place in landfills. To protect the groundwater from contamination by landfill leachate, it is quite essential to provide the bottom barrier of suitable thickness, and to minimize the amount of water that could enter the landfill to create leachate. The present study was undertaken to determine the rate of movement of potential contaminants from its bottom to the aquifer media, so as to evolve a rational method for the determination of thickness of bottom barrier on sound engineering practice, in place of adopting a prescriptive standard, which is very common in most of the Asian countries. The study was undertaken for non conservative contaminant Dissolved Organic Carbon (DOC). The governing equation of contaminant transport was solved using finite difference method, and finite mass boundary condition to ape the finite mass of contaminants in a landfill. The solution of the model was run in MatLab 7.0 for a range of Darcy velocities and retardation factors for a representative equivalent height of leachate. Design curves were drawn which can be used for determination of suitable barrier thickness on the basis of expected maximum concentration of DOC in landfill leachate and maximum permissible concentration of the same.
\end{abstract}

Keywords: Landfill, groundwater, contaminant transport, barrier thickness.

\section{BACKGROUND}

A sanitary landfill is defined as a system in which municipal solid wastes are disposed of, compacted, and covered with a layer of soil at the end of each day's operation [1]. However, poorly designed landfills can create contamination of groundwater, soil, and air. The most commonly reported danger to the human health from these landfills is from the use of groundwater that has been contaminated by leachate [2-4]. As water percolates through the landfill, contaminants are leached out from the solid waste. Leachate is produced when moisture enters the refuse in a landfill, extracts contaminants into the liquid phase, and produces moisture content sufficiently high to initiate liquid flow. Leachate may contain dissolved or suspended material associated with wastes disposed off in the landfill, as well as many byproducts of chemical and biochemical reactions. Leachate tends to migrate in surrounding soil and may ultimately result in contamination of underlying soil and groundwater. The rate and characteristics of leachate produced depends on many factors such as solid waste composition, particle size, degree of compaction, hydrology of site, age of landfill, moisture and temperature conditions, and available oxygen. During

Address correspondence to this author at the Department of Civil \& Environmental Engineering, Delhi college of Engineering, Delhi, India; Tel: 1111-27871012; E-mail: madhyabharat@rediffmail.com the course of stabilization of landfilled wastes, nonconservative constituents of leachate (primarily organic in nature) tend to decompose and stabilize with time, whereas conservative constituents will remain long after waste stabilization occurs. Conservative constituents include various heavy metals, chloride, and sulfide. The concentration of non conservative contaminants in the landfill leachate increases in the beginning, reaches a peak, declines thereafter [5-6].

The containment systems for modern sanitary landfills involve the use of barrier layers called liner to prevent leachate from leaving the landfill and contaminating the underlying soil and groundwater. Landfill design requirements include provisions of bottom liners of compacted clays and/or compacted clays coupled with geosynthetic materials in suitable thickness to contain or retard the migration of landfill leachate to the underlying aquifer. Clay happens to be the material of choice for waste containment because it provides sufficient impermeability. The current design of landfill bottom barrier is based upon the type of waste to be disposed, the geography of site, material standards, and regulatory requirements.

\section{MODELING OF CONTAMINANT TRANSPORT PROCESSES}

Exposures to toxic environmental contaminants are significant risk factors in human health and disease. To understand and manage these risk factors, environmental and pub- 
lic health managers must have knowledge of the source of the exposure, the fate of contaminants, exposure levels, and routes of the exposure for such contaminants. Environmental fate, transformation, and transport models are used to assist in understanding, relating, and quantifying such contamination from the sources. The process of groundwater flow is generally assumed to be governed by Darcy's law and the conservation of mass. The theoretical basis for the equation describing solute transport has been well documented in the literature [7-10]. Reilly et al. [11] provide a conceptual framework for analyzing and modeling physical solute transport processes in groundwater.

Changes in chemical concentration occur within a dynamic groundwater system primarily due to four distinct processes viz. (i)advective transport, which involves moving of the contaminants with flowing groundwater (ii)diffusive transport, which involves the movement of contaminant due to concentration gradient (iii)dispersion, which involves mixing of the contaminants at relatively high flows due to local variations in the flow velocity of groundwater, or mixing and spreading of contaminants in the form of dissolved molecules and ions due to variations in flow velocity through porous media resulting from non-homogeneity of aquifer (iv)reaction, absorption, or decay of the contaminants. Thus, when dealing with contaminants in groundwater, the mass of contaminant transported by advection per unit area per unit time is expressed as $f=n v c=v_{a} c$, where $n$ is the effective porosity of soil, $v$ is groundwater velocity (seepage velocity), $v_{a}$ is Darcy velocity, $c$ is concentration of contaminant at the point and time of interest. Diffusion involves the movement of contaminant from points of high chemical potential (concentration) to points of low chemical potential (concentration). When contaminant migration is associated with relatively high flows (as in many aquifers), there is a third transport mechanism to be considered, viz. mechanical dispersion. Mechanical dispersion involves mixing that occurs due to local variations in the flow velocity of the groundwater. The dispersion of contaminants also involves a mixing and spreading of the contaminants due to non-homogeneity in the aquifer. Although, this mechanism is totally different from the diffusion process, for most practical applications, it can be mathematically modeled in the same way, hence the two processes are often lumped together as composite parameter $D_{h}$, called the coefficient of hydrodynamic dispersion expressed as $D_{h}=D_{e}+D_{m d}$, where $D_{e}$ is the effective molecular diffusion coefficient for the contaminant species of interest, $D_{m d}$ is the coefficient of mechanical dispersion. When dealing with transport through intact clayey soil, diffusion will usually control the parameter $D_{h}$ and dispersion is negligible. In aquifers, the opposite tends to be true and dispersion tends to dominate. It is often convenient to model the dispersive process as linear function of velocity. Thus $D_{m d}=\alpha v$, where $\alpha$ is dispersivity. The dispersivity tends to be scale dependant. Effective molecular diffusion coefficient in soil $D_{e}$ tends to be smaller than the molecular diffusion coefficient $D_{m}$, because the diffusing substance must follow a tortuous path between two points through the porous medium rather than a straight one. Effective molecular diffusion coefficient is thus expressed as $D_{e}=\tau D_{m}$. Millington and Quirk [12] present a theory suggesting a relationship between tortuosity of the medium $\tau$ and porosity $n$ as $\tau=n^{1 / 3}$. Thus for a medium with porosity $n=0.4$, tortuosity tends to be equal to 0.74 . Mathematically, advective-dispersive transport in one dimension can be expressed as:

$$
f_{T}(c, \tau)=n v_{x} c-n D_{h} \frac{\partial c}{\partial z}
$$

Where $n$ is effective porosity of soil, $D_{h}$ is the hydrodynamic dispersion coefficient, $\partial \mathrm{c} / \partial \mathrm{z}$ is the concentration gradient, $v_{x}$ is advective velocity, and $c$ is the concentration of contaminant in source. The negative sign arises from the fact that the contaminant move from high to low concentrations. The total mass of contaminant transported out of landfill up to some specific time $t$, is obtained by integrating the above equation.

Governing equation for the transport of contaminant through porous media is expressed by the equation (2) which simply says that the increase in contaminant concentration within a small region is equal to the increase in mass due to advective-diffusive transport minus the decrease in mass due to sorption minus the decrease in mass due to first order decay processes.

$$
\frac{\partial C}{\partial t}=\frac{D_{h}}{R_{f}} \frac{\partial^{2} C}{\partial z^{2}}-\frac{v}{R_{f}} \frac{\partial C}{\partial z}
$$

Where $n$ is the effective porosity of soil, $C$ is the concentration at depth $z$ and time $t, D_{h}=D_{e}+D_{m d}$ is the coefficient of hydrodynamic dispersion, $v$ is advective velocity, $R_{f}=1+\rho K_{d} / n$, is the retardation factor which is supposed to represent the lumped behavior of absorption and decay process. The term $K_{d}$ is obtained from batch absorption isotherms, and $\rho$ is mass density of soil.

\section{BOUNDARY CONDITIONS}

The governing equation (1) alone is not sufficient to describe a specific physical system. This is because a general solution of $n^{\text {th }}$ order differential equation will involve $n$ independent arbitrary constants or functions. In order to define uniquely a given physical problem, the values of constants or forms of the functions must be specified. Initial and boundary conditions can be used to provide this required additional information. Generally, boundary conditions specify the value of dependent variable, or the value of the first derivative of the dependent variable, along the boundaries of the system being modeled.

Initial and boundary conditions provide the additional information required to obtain unique solution to governing partial differential equation. For steady-state problems, only boundary conditions are required, whereas for transient problems, both boundary and initial conditions must be specified. Mathematically, the boundary conditions include the geometry of the boundary and the values of the dependent variable or its derivative normal to the boundary. Boundary conditions are typically derived from physical and/or hydraulic boundaries of system.

In general three kinds of boundary conditions may be applied to the governing equation of contaminant transport. The first type of boundary condition, also called Dirichlet 
condition, specifies the value of primary variable $\mathrm{C}$ (concentration of the contaminant) at a boundary such that

$$
\begin{array}{lll}
C\left(x_{0}, t\right)=C_{0} & \text { or } & C\left(x_{0}, t\right)=C_{0}(t) \\
C\left(x_{L}, t\right)=C_{L} & \text { or } & C\left(x_{L}, t\right)=C_{L}(t) \ldots \ldots
\end{array}
$$

In the contaminant transport problem, a constant or time dependent concentration when taken as a boundary condition constitutes Dirichlet, or first type of boundary condition. A constant concentration of inflowing solution as a boundary condition is maintained in some experimental studies to determine the parameters. However a time dependent concentration is taken as a boundary condition in landfill modeling where the seasonal variation of concentration can be determined by analyzing the extracted pore water from the landfill. The method of measuring and analyzing the pore water at different depths was also suggested by Johnson et al. [13].

The second type of boundary condition is referred to as Neumann or flux condition, and it specifies the flux (fluid flux) across an area. The term flux means the mass of contaminant per unit time passing through unit surface area. This condition is expressed as

$$
\begin{aligned}
& -\left.K \frac{d C}{d x}\right|_{x=x_{0}}=q_{0} \text { or } \\
& -\left.K \frac{d C}{d x}\right|_{x=x_{L}}=q_{L}
\end{aligned}
$$

In the contaminant transport equation, this boundary condition is used when there is an impermeable layer at the bottom of the domain. Similarly in most of the analytical models the boundary condition at the end of the infinite domain is also modeled by taking $\frac{\partial C}{\partial x}=0$. The same condition prevails when the contaminant moves out of soil with moving soil water, however dispersion and diffusion do not contribute to this movement.

The third type of boundary condition called Cauchy condition, prescribes a linear combination of $C$ and the flux at a boundary such that,

$$
\begin{aligned}
& {\left[a_{0} C+b_{0} K \frac{d C}{d x}\right]_{x=x_{0}}=v_{0} \text { and }} \\
& {\left[a_{L} C+b_{L} K \frac{d C}{d x}\right]_{x=x_{L}}=v_{L}}
\end{aligned}
$$

Where $a_{0}, b_{o}, a_{L}$, and $b_{L}$ are the known coefficients. The amount of contaminants entering the soil depends upon the flux of the water into the soil. The concentration flux boundary condition also belongs to this category. Cauchy boundary condition is summation of two components of contaminant flux, one being constant, and other being function of time.

The selection of boundary condition is a critically important step for the conceptualizing and developing a model for a contaminant transport problem. Even if one of the bound- ary condition is inappropriate for a defined contaminant transport problem, it is most likely that the final expression will be erroneous or will produce ambiguous results.

\section{FINITE MASS OF CONTAMINANT}

The mass of contaminant within a landfill is limited and mass will be reduced as the contaminant is transported into soil. The concentration of contaminants in landfill leachate increases during the operation of landfill facility, reaches a peak, and then declines. The increase in concentration is related to physical processes of leaching of contaminants, chemical and biological processes which generate chemical species of interest from the synthesis, or breakdown of existing species. Likewise, the decrease in concentration with time may be related to physical process of removal of contaminants in the form of leachate from the landfill, chemical and biological processes which result in precipitation and/or synthesis or breakdown of the chemical species of interest into other chemical forms. In the design of barrier systems it is generally not practical to model the details of leaching processes and reasonable engineering approximations can be made to model the impact of finite mass of contaminant. Thus for the purposes of performing design calculations, it is often conservative to assume, that the concentration of contaminant of interest reaches a peak, $\mathrm{c}_{0}$, instantaneously, and all of the mass of contaminant species $m_{t c}$ is in solution the time when peak concentration occurs. The mass of the contaminant available for transport into the soil can be represented in terms of the peak concentration, $c_{0}$, and the reference height of leachate, $H_{r}$, or the equivalent height of leachate $H_{f} . H_{r}$, may be defined for each contaminant species of interest and corresponds to the volume of fluid (per unit area of landfill) that, at a concentration $c_{0}$, would contain the total mass $m_{0}$, of that contaminant species which could be released either for transport or collection. The equivalent height of leachate $H_{f}$, corresponds to that portion of mass that is available for transport into hydro geologic system. It does not include the mass of contaminant which exists or is expected to exist in solid/immobile form, or contaminant that is released in gas phase. Thus, the essential difference between $H_{r}$ and $H_{f}$ is that the $H_{r}$ includes the mass collected by the leachate collection system, whereas $H_{f}$ excludes this mass. If the portion of the mass of contaminant collected by or removed by leachate collection system is ignored, variation in the concentration of contaminant in source at the top of barrier can be written as [14],

$$
C_{T}=C_{0}-\frac{1}{H_{f}} \int_{0}^{t} f_{T}(c, \tau) d \tau
$$

Where $C_{T}$ is the concentration of contaminant in source at any time $t, C_{0}$ is the initial concentration of contaminant in source, $H_{f}$, is the equivalent height of leachate. Integration term on the right hand side of equation indicates cumulative mass of contaminant moved out from the source into soil. Substituting for mass flux $f_{T}(c, \tau)$ from equation (1), equation (6) can be written in numerical form as

$$
C_{T}(0, t)=C_{0}-\frac{n}{H_{f}}\left(v_{x} \sum_{t=0}^{t} C_{T}(0, t) \Delta t-D_{h} \sum_{t=0}^{t} \Phi(0, t) \Delta t\right)
$$


Where the term $\varphi(0, t)$ stands for $\partial \mathrm{c} / \partial \mathrm{z}$ at the top of barrier at any time $t$.

\section{MATERIALS AND METHODS}

Governing one dimensional mass transport equation based on advection dispersion processes represented by equation (2) was solved using Matlab 7.0 and finite difference approach with upwind correction. Thus equation (2) in finite difference form with upwind correction can be written as [10]

$$
\begin{aligned}
& \frac{C_{i}^{m+1}-C_{i}^{m}}{\Delta t}+\frac{1}{R_{f}} v_{x} \frac{C_{i}^{m}-C_{i-1}^{m}}{\Delta x}- \\
& \frac{1}{R_{f}} D_{x} \frac{C_{i+1}^{m}-2 C_{i}^{m}+C_{i-1}^{m}}{(\Delta x)^{2}}=0
\end{aligned}
$$

Writing $\kappa_{1}=\frac{1}{R_{f}} \frac{v_{x} \Delta t}{2 \Delta x}$ and $\kappa_{2}=\frac{1}{R_{f}} \frac{D_{x} \Delta t}{(\Delta x)^{2}}$, and

rearranging the terms in equation (8), yields

$$
\begin{aligned}
& C_{i}^{m+1}=C_{i}^{m}-\left(1-2 \kappa_{1}-2 \kappa_{2}\right) C_{i}^{m}+ \\
& \kappa_{2} C_{i+1}^{m}+\left(2 \kappa_{1}+\kappa_{2}\right) C_{i-1}^{m}=0
\end{aligned}
$$

The method is implemented by marching the solution forward at the interior nodes, bringing with it the effects of the initial condition and the boundary nodes. For the implementation of the above solution in MatLab, the domain of problem is discritized in suitable number of nodes for time and space, in such a way that $\frac{1}{R_{f}} \frac{D_{x} \Delta t}{(\Delta x)^{2}}+\frac{1}{R_{f}} \frac{v_{x} \Delta t}{2 \Delta x} \leq \frac{1}{2}$.

Thus, the entire domain shall be divided in say, Ntsteps $=\mathrm{T} / \Delta t$, and $\mathrm{N} x$ steps $=\mathrm{z} / \Delta x$. Initial and boundary conditions are implemented by keeping $C_{i}^{m}$ at the beginning of solution to be zero everywhere along the entire depth of domain. Thus, for the implementation of initial condition, keeping $C_{i}^{m}$ to be zero everywhere,

$$
C_{i}^{m}=C_{2}^{m}=C_{3}^{m}=C_{4}^{m}=C_{5}^{m}=\ldots \ldots \ldots \ldots \ldots . . .=C_{\text {Nxsteps }}^{m}
$$

Implementation of the solution using finite difference method is illustrated in Fig. (1).

\section{MODEL VALIDATION FOR DOC TRANSPORT}

Model developed herein was tested for the field data of transport of DOC. Field data of Munro et al. [15] for a landfill at New Brunswick was used to validate the numerical model. The field profiles of DOC in bore hole number L1B was compared with simulation results of the model solution. In their investigation, the hydraulic gradients in the till underlying the landfill waste was determined by them using data from monitoring wells, and from comparison of the values obtained for horizontal and vertical hydraulic gradients. It was concluded by them, that the movement of contaminant was predominantly vertical. For calibrating field data they adopted a process based approach for estimating the temporal concentrations of DOC at the waste till interface. This concentration in turn was adopted as the source concentration (input) in their mathematical model. DOC concentration at the influent boundary condition was thus not constant but varying with respect to the duration of monitoring ( 6.75 years). The variation of DOC concentration at influent boundary condition is shown as histogram of DOC concentration in Fig. (2). Due to the possible uncertainties in the DOC source function, comparison of the field data and numerical results was considered to be good if the model simulation adequately fit the steeply declining concentrations in the top one meter of the profile. For greater depths (more than $1 \mathrm{~m}$ below the landfill), their model results did not agree with the observed data. The field parameters obtained by Munro et al. are shown in Table 1, and the simulated and observed field concentration of DOC is shown in Table $\mathbf{2}$.

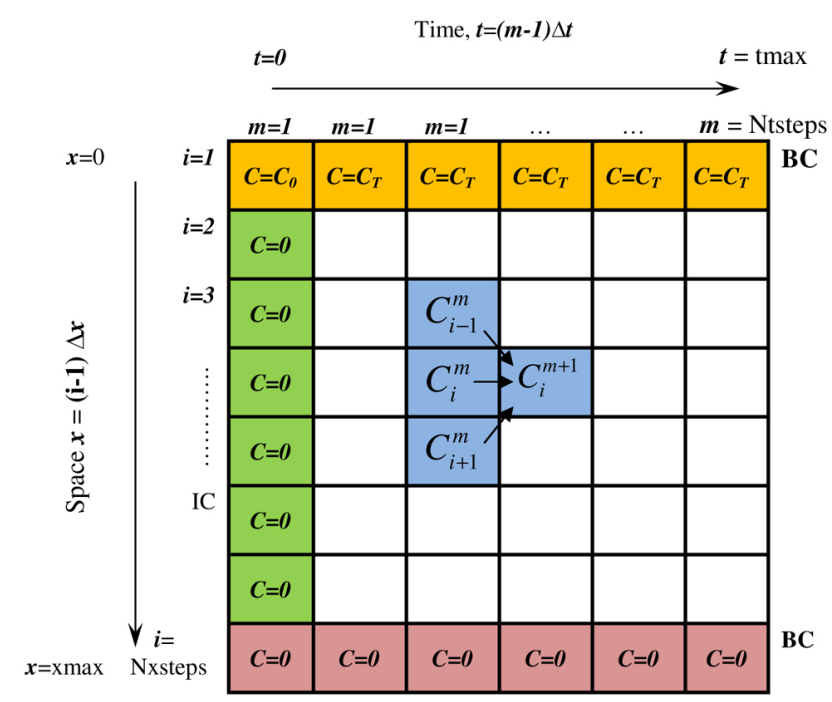

Fig. (1). Schematic of solution using finite difference method and MatLab.

Table 1. Model Parameters, Munro, DOC

\begin{tabular}{|c|c|c|c|}
\hline S.No. & Model Parameter & Unit & Value \\
\hline \hline 1 & Depth & $\mathrm{m}$ & 4.0 \\
\hline 2 & Effective Molecular Diffusion & $\mathrm{m}^{2} / \mathrm{yr}$ & 0.02 \\
\hline 3 & Retardation factor (R) & & 1.5 \\
\hline 4 & Average Linear Velocity & $\mathrm{m} / \mathrm{yr}$ & 0.7 \\
\hline 5 & Dispersivity & $\mathrm{m}$ & 0.05 \\
\hline 6 & Porosity & & 0.28 \\
\hline
\end{tabular}

\section{DESIGN CHARTS FOR BARRIER THICKNESS}

In the design of a landfill system, it is quite common to design a landfill site for a maximum period of 50 years in which the first 10-15 years are for waste receiving and the remaining for post-closure [16-17]. Thus, it is logical and reasonable to set the required time of travel, $t$, of a contaminant through a liner equal to the design life of a landfill (50 
Table 2. Simulated and Observed DOC Concentration Profile at Bore Hole L1B

\begin{tabular}{|c|c|c|}
\hline Depth (m) & $\begin{array}{c}\text { After 6.75 Years } \\
(\text { Munro et al. } \mathbf{~ 1 9 9 7} \\
\mathbf{m g} / \mathbf{L})\end{array}$ & $\begin{array}{c}\text { After 6.75 Years (Simu- } \\
\text { lated) }\end{array}$ \\
\hline 0.16 & 10000 & 12670 \\
\hline 0.3 & 8040 & 10444 \\
\hline 0.4 & 5000 & 7075 \\
\hline 0.54 & 4200 & 4585 \\
\hline 0.65 & 3700 & 3436 \\
\hline 0.76 & 2850 & 2886 \\
\hline 0.82 & 2760 & 2790 \\
\hline 1.08 & 2860 & 2552 \\
\hline
\end{tabular}

\section{Histogram : DOC Concentration}

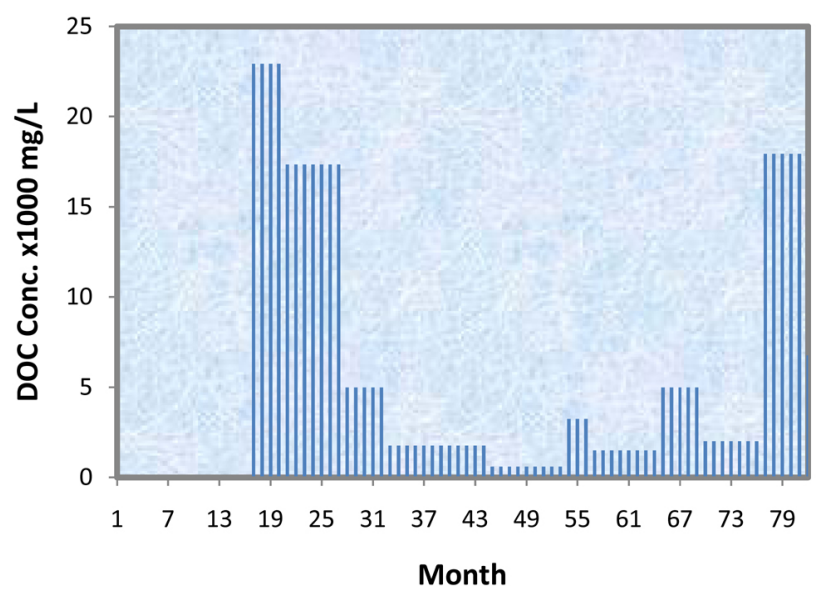

Fig. (2). Histogram : DOC concentration.

years). For a landfill liner with a given set of liner properties e.g., hydraulic conductivity, porosity and transport parameters e.g., diffusion coefficient and retardation factor as determined by laboratory column and batch sorption tests, a design chart can be plotted which will show the change in relative concentration of solute with respect to depth at a certain period of time. Such chart can be utilized for the determination of liner thickness if the maximum permissible relative concentration after a certain period of time is defined based on pollution prevention criteria in a region. DOC transport from landfills was studied for a period of 50 years for the range of parameters as listed in Table $\mathbf{3 A}$ and Table 3B.
Table 3A. Common Data for Simulation (Chloride)

\begin{tabular}{|c|c|c|}
\hline S. No. & Parameter & Value \\
\hline \hline 1 & Duration & 50 years \\
\hline 2 & Porosity & 0.40 \\
\hline 3 & $\begin{array}{c}\text { Effective Molecular Diffu- } \\
\text { sion Coefficient } D_{e} \\
=\tau D_{m}\end{array}$ & $0.02 \mathrm{~m}^{2} /$ year \\
\hline 4 & Retardation Factor $\left(R_{f}\right)$ & $1,3,12,24,100$ \\
\hline
\end{tabular}

Table 3B. Simulation Data for Barrier Thickness

\begin{tabular}{|c|c|c|c|}
\hline S. No. & $\begin{array}{c}\text { Advective } \\
\text { Velocity } \\
\boldsymbol{v}_{\boldsymbol{x}}=\mathbf{v} / \boldsymbol{n} \\
(\mathbf{m} / \mathbf{y r})\end{array}$ & $\begin{array}{c}\text { Dispersivity, } \mathbf{a}_{\mathbf{L}} \\
(\mathbf{m})\end{array}$ & $\begin{array}{c}\text { Hydrodynamic Disper- } \\
\text { sion Coefficient } \boldsymbol{D}_{\boldsymbol{h}}= \\
\boldsymbol{\tau} \boldsymbol{D}_{\boldsymbol{m}}+\mathbf{a}_{\mathbf{L}} \cdot \boldsymbol{v}_{\boldsymbol{x}}\end{array}$ \\
\hline \hline 1 & 0.00075 & 0.01 & 0.014803 \\
\hline 2 & 0.0075 & 0.03 & 0.01489 \\
\hline 3 & 0.075 & 0.05 & 0.0163 \\
\hline 4 & 0.15 & 0.10 & 0.0208 \\
\hline 5 & 0.25 & 0.14 & 0.0288 \\
\hline
\end{tabular}

The range of Darcy velocity for Municipal Landfill has been found to be in the range of $0.0003 \mathrm{~m} / \mathrm{yr}$ to $0.03 \mathrm{~m} / \mathrm{yr}$ for compacted liners, and $0.03 \mathrm{~m} / \mathrm{yr}$ to $0.1 \mathrm{~m} / \mathrm{yr}$ for aquitards [18-20]. Also, at low velocities for most of the contaminants found in municipal landfills (Rowe et al.), the computed retardation factor assumes a value ranging from 1 to 100 . The simulation was run for finite mass of the contaminant as the upper boundary, where the initial concentration of contaminant in leachate was taken as $1000 \mathrm{mg} / \mathrm{L}$ and the porosity of barrier/aquitard was assumed as 0.4 , with equivalent height of leachate taken as $10 \mathrm{~m}$. The model gives the spatial and temporal variation of DOC concentration along the thickness of the barrier and the maximum concentration recorded in each depth for the entire domain (50 Years). The simulation was run for time domain of 50 years, and the time step was determined after satisfying the stability criteria of solution. The time step was thus in the range of 0.04 year to 0.25 year for the element sizes ranging from $0.06 \mathrm{~m}$ to $0.08 \mathrm{~m}$. The initial concentration (background) was assumed to be zero for a fully flushed boundary. The non conservative contaminant front in all the cases could not reach the bottom boundary for the cases of both, small and large Darcy velocities. The maximum concentration attained at various depths for a number of leachate heights was determined next.

\section{RESULTS AND DISCUSSIONS}

From the results plotted graphically (Figs. 3-7), one can determine the minimum design thickness of barrier knowing the permissible concentration of that particular contaminant. If the percentage of contaminant species of interest present in waste is known through chemical analysis or through typical landfill data charts, and the total design weight in metric tons is available, then the total mass of the contaminant present in waste can be computed. Knowing the quantity of waste that 


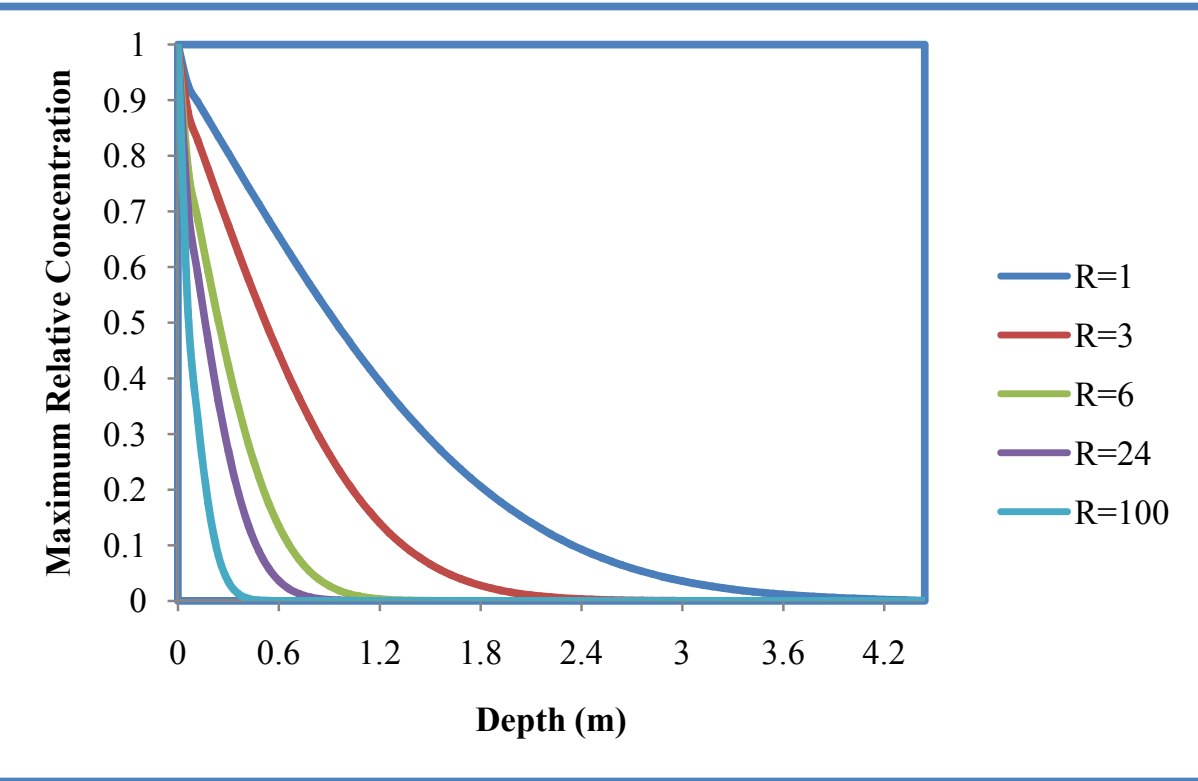

Fig. (3). Variation maximium relative conentration $(\mathrm{DOC})$ darcy velocity $=0.03 \mathrm{~cm} / \mathrm{yr}$, Time $=50$ years, $\mathrm{Hf}=10 \mathrm{~m}$.

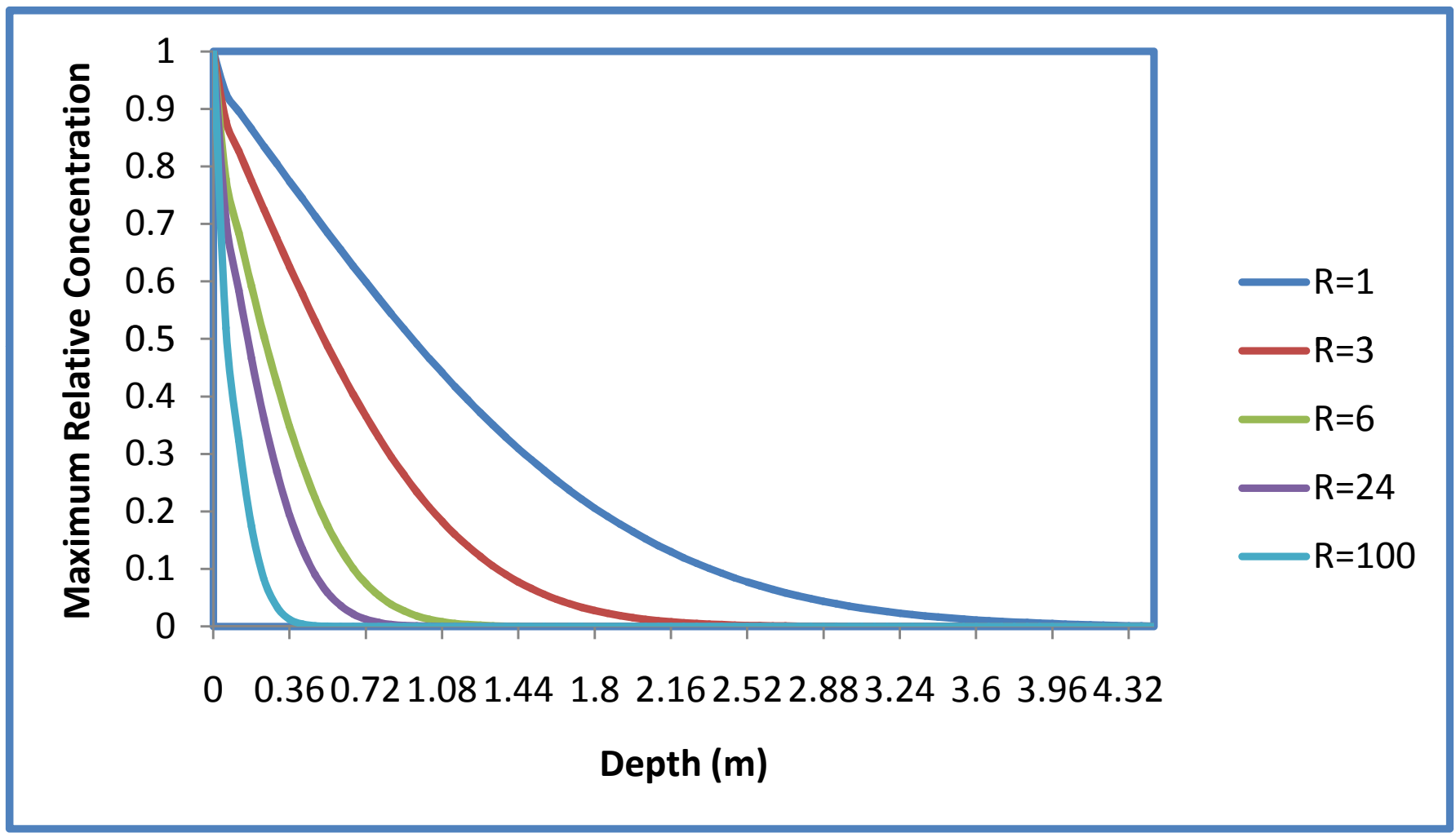

Fig. (4). Variation of maximum relative concentration $(\mathrm{DOC})$ darcy velocity $=0.3 / \mathrm{cm} / \mathrm{yr}$, Time $=50$ years, $\mathrm{Hf}=10 \mathrm{~m}$. 


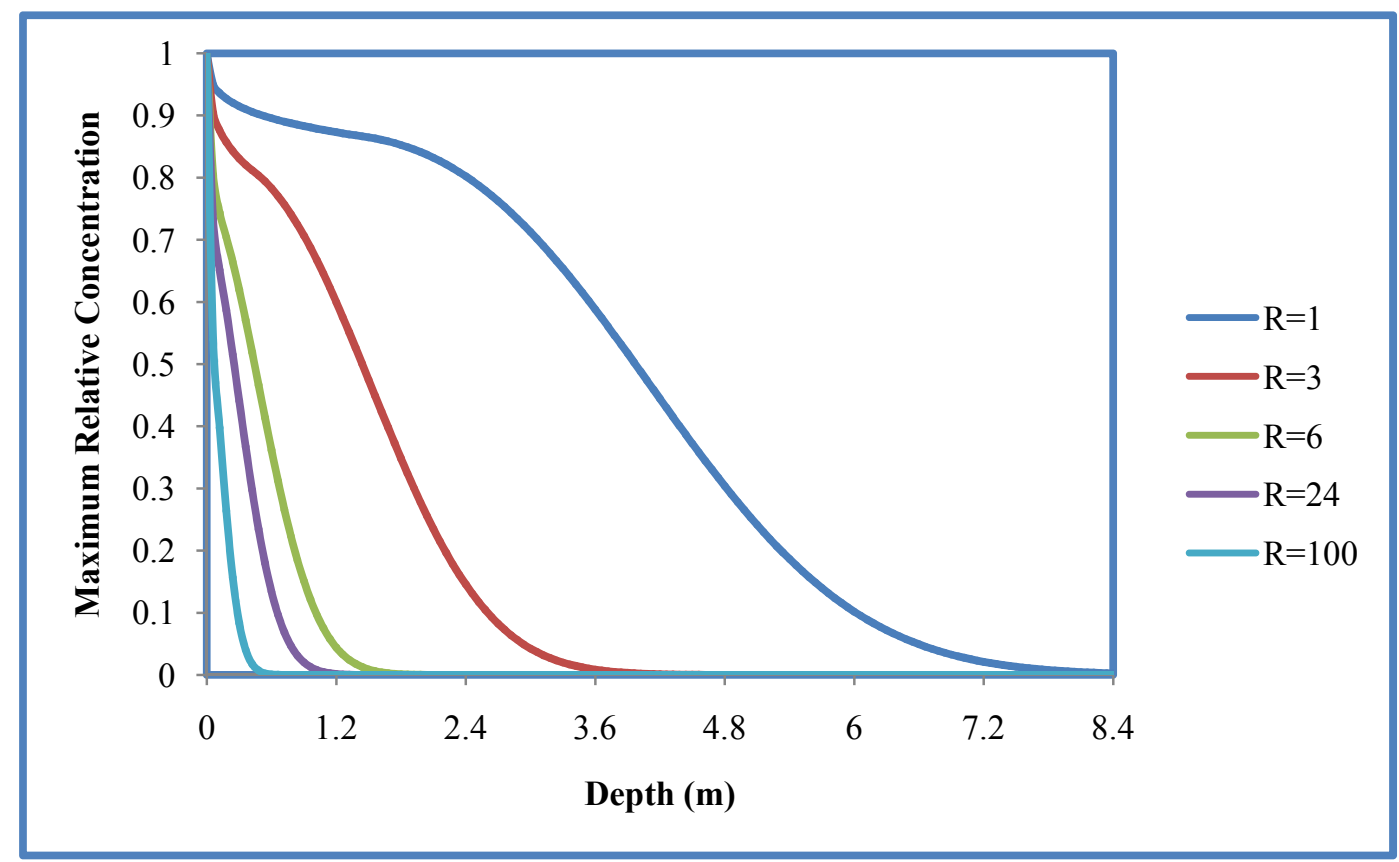

Fig. (5). Variation of maximum relative concentration (DOC) darcy velocity $=3 \mathrm{~cm} / \mathrm{yr}$, Time $=50$ years, $\mathrm{Hf}=10 \mathrm{~m}$.

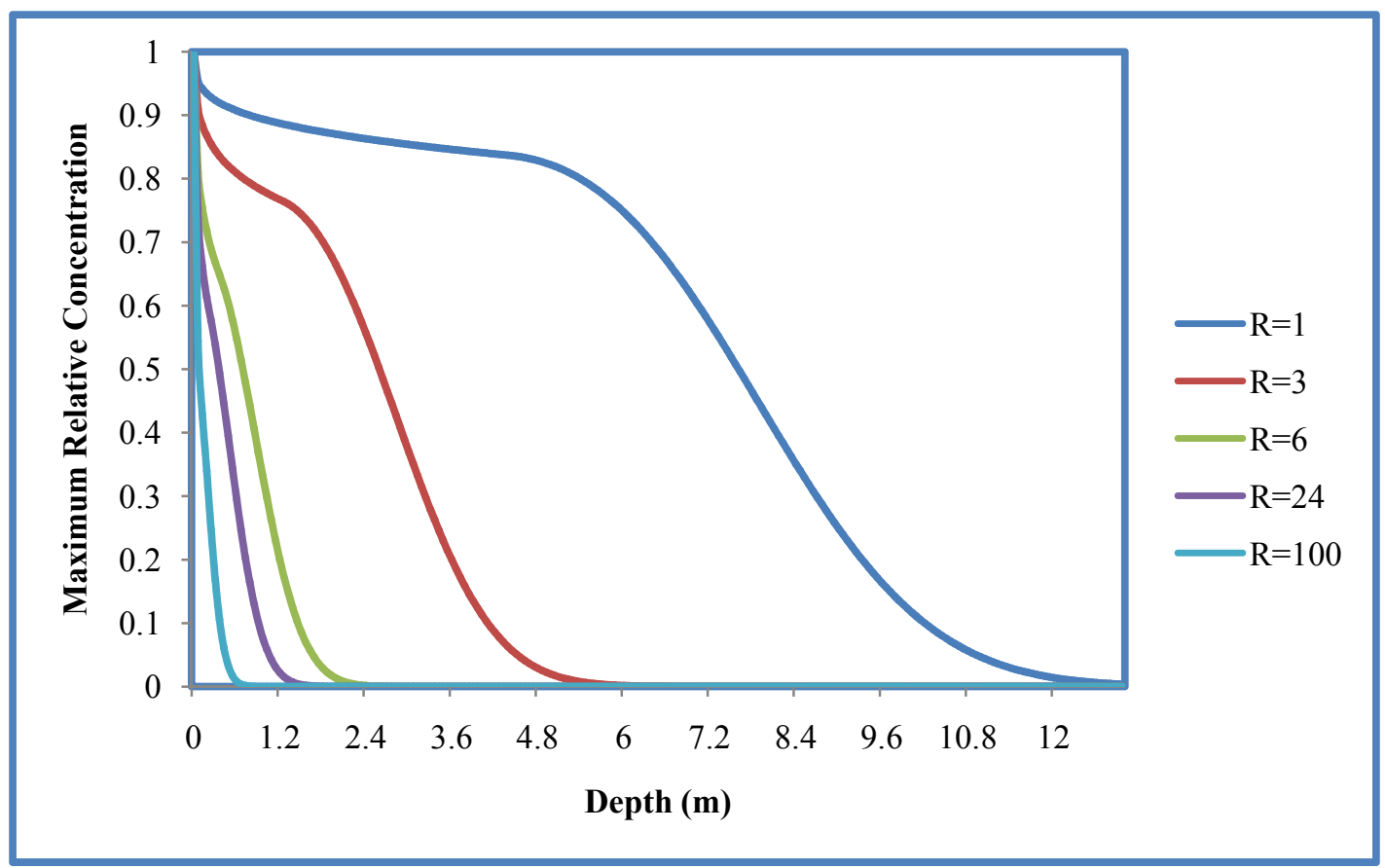

Fig. (6). Variation of maximum relative concentration $(\mathrm{DOC})$ darcy velocity $=6 \mathrm{~cm} / \mathrm{yr}$, Time $=50$ years, $\mathrm{Hf}=10 \mathrm{~m}$. 


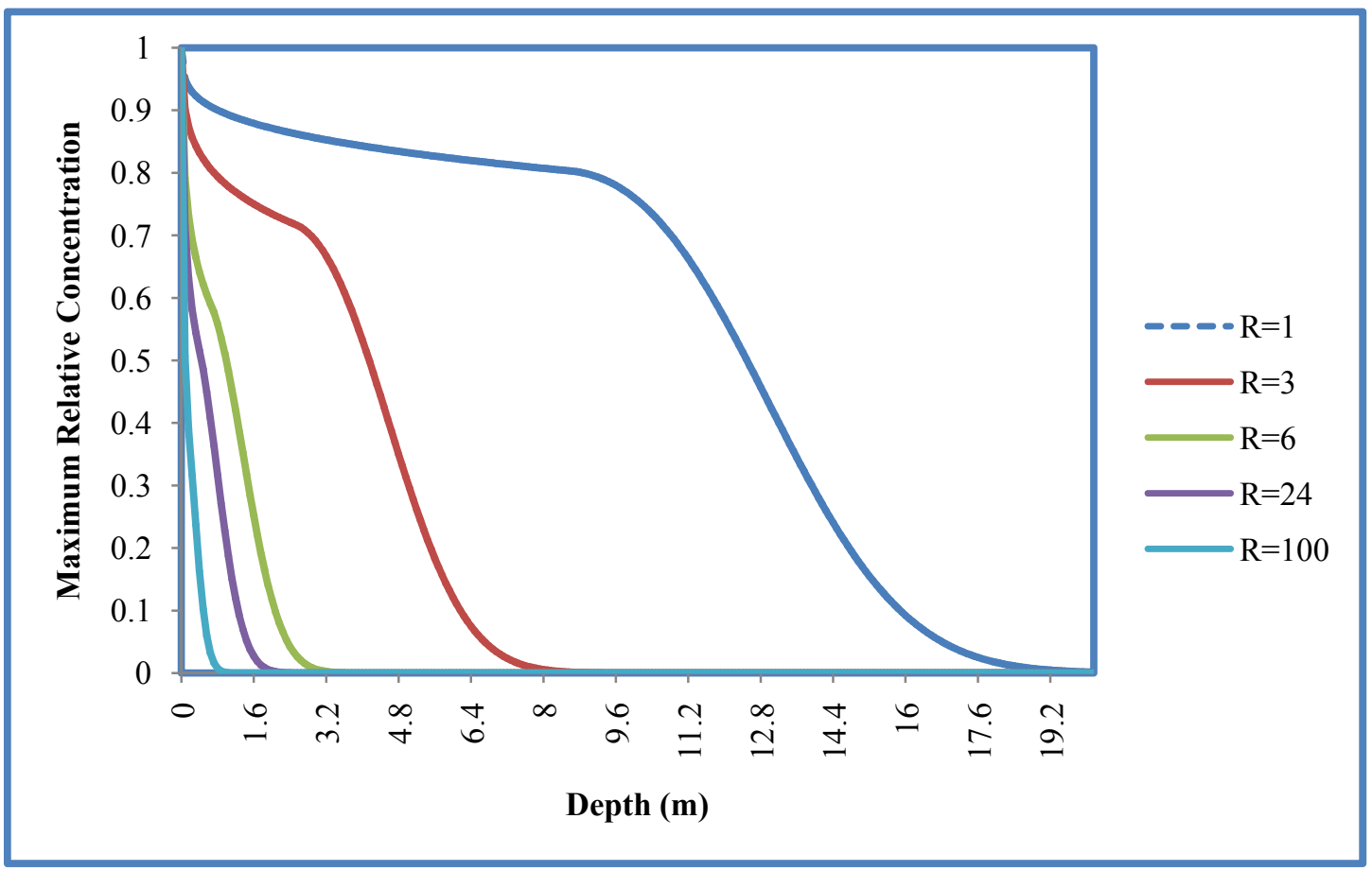

Fig. (7). Variation of maximum relative concentration $(\mathrm{DOC})$ darcy velocity $=10 \mathrm{~cm} / \mathrm{yr}$, Time $=50$ years, $\mathrm{Hf}=10 \mathrm{~m}$.

infiltrates through the bottom of the waste into the barrier, and aerial extent of landfill, the equivalent leachate height of the landfill can be determined. Minimum thickness of liner can be determined with the known value of maximum permissible concentration of contaminant species of interest as per regulatory criteria. The permissible value when divided by the typical maximum concentration of that contaminant in the landfill leachate gives the design maximum relative concentration. Equivalent height of leachate can be determined by dividing the total mass of contaminant present in waste by typical maximum concentration of that contaminant in landfill leachate. Appropriate minimum thickness of barrier for a known value of equivalent height of leachate, and design maximum relative concentration can be determined from these figures. Retardation coefficient value $R$, has to be computed on the basis of batch absorption tests, if the bottom barrier provided is expected to have enhanced absorption capacity for organic contaminants. For the case, when compacted clays are to be used for providing a bottom barrier, the value of retardation coefficient $R$, may be taken as 1 . On comparing Figs. (3-7), one observes that there is hardly any variation in the respective design charts for the velocities $0.0003 \mathrm{~m} / \mathrm{yr}$ and $0.003 \mathrm{~m} / \mathrm{yr}$ respectively. This implies that the advection is not the primary transport mechanisms at such low velocities and that the contaminant transport takes place on account of diffusion only. As the Darcy velocity increases tenfold, the minimum barrier thickness required also increases. In case where there is no leachate collection system, the value of equivalent leachate height is large. From the results plotted one can see that maximum depth travelled to reach a maximum relative concentration of zero, at $\mathrm{R}$ value of 3 , increases from about $3 \mathrm{~m}$ for Darcy velocity of $0.03 \mathrm{~cm} / \mathrm{yr}$ to $8 \mathrm{~m}$ for Darcy velocity of $10 \mathrm{~cm} / \mathrm{yr}$. For $\mathrm{R}$ value of 100 , the maximum depth required to reach maximum relative concentration of zero at highest Darcy velocity considered does not exceed about $1 \mathrm{~m}$.

\section{CONCLUSIONS}

Mass transport of organic contaminants from landfill leachate was modeled taking into account the mechanisms of contaminant transport viz. advection and dispersion, and using the finite difference method with upwind correction. The model was solved in MatLab 7.0 and validated with field data of contaminant transport (Munro et al. 1997). Simulation of validated model were run for a range of parameters commonly observed in municipal solid waste disposal sites for a duration of 50 years. Design charts were drawn from the results of such simulation giving maximum relative concentration with depth at the end of simulation period over the domain of interest. Transport of contaminants was subjected to finite mass of contaminant boundary condition to ape the field conditions of Landfill. Design charts prepared can be used for determining the minimum thickness of barriers required at the bottom of landfill so as to meet the regulatory requirements of organic contaminant containment effectively.

\section{REFERENCES}

[1] Tchobanoglous G, Theisen H, Eliassen R. Solid Wastes Engineering - Principles and Management Issues. McGraw Hill, New York 1997.

[2] Chian ESK, DeWalle FB. Sanitary landfill leachates and their treatment. J Environ Eng Div (ASCE) 1976; 102(2): 411-31.

[3] Kelley WE. Groundwater pollution near a landfill. J Environ Eng Div (ASCE) 1976; 102(6): 1189-99.

[4] Lo IMC. Characteristics and treatment of leachates from domestic landfills. Environ Int 1996; 22(4): 433-42. 
[5] Kouzeli-Katsiri A, Bosdogianni A, Christoulas D. Prediction of leachate quality from sanitary landfills. J Environ Eng (ASCE) 1999; 125(10): 950-8.

[6] Farquhar GJ. Leachate: Production and characterization. Can J Civil Eng 1989; 16: 317-25.

[7] Bear J, Sorek S, Borisov V. Eulerian- lagrangian formulation of balance equations in porous media. Num Methods Partial Differ Equations 1995; 13(5): 505-30.

[8] Domenico PA, Schwartz FW. Physical and Chemical Hydrogeology. 2nd ed. John Wiley \& Sons: New York 1998; p. 506.

[9] Sudicky EA. One-Dimensional Advective-Dispersive Solute Transport Modeling in Semi-Infinite Domain with Time-Variable Concentration Input at Source Computer Software Documentation. Waterloo Centre for Groundwater Research, University of waterloo, Waterloo, Ontario 1998.

[10] Zheng C, Bennett GD. Applied Contaminant Transport Modeling. Van Norstrand Reinhold, New York 1995.

[11] Reilly TE, Franke OL, Buxton HT, Bennett GD. A Conceptual Framework for Groundwater Solute Transport Studies with Emphasis on Physical Mechanisms of Solute Movement. U.S. Geological Survey Water-Res. Inv. Rept1987.

[12] Millington RJ, Quirk JM. Permeability of porous solids. Trans Faraday Soc 1961; 57: 1200-7.
[13] Johnson RL, Cherry JA, Pankow JF. Diffusive contaminant transport in natural clay: a field example and implication for clay lined waste disposal site. Environ Sci Technol Am Chem Soc 1989; 23: $340-9$.

[14] Rowe Kerry R, Quigley Robert M, Booker John R. Clayey Systems for Waste Disposal Facilities. E \& FN Spon, UK 1995; p. 390.

[15] Munro IRP, MacQuarrie KTB, Valsangkar AJ, Kan KT. Migration of landfill leachate into a shallow clayey till in southern new brunswick: a field and modeling investigation. Can Geotech J 1997; 34: 204-19.

[16] Rowe RK. The role of modeling in the design of barriers. Can Geotech J 1988; 25: 778-98.

[17] Swarbrick GE. Current understanding of the physical properties of the landfill. Chem Eng Aust 1994; 19: 18-23.

[18] Rowe RK, Nadarajah P. Evaluation of the hydraulic conductivity of aquitards. Can Geotech J 1993; 30: 781-800.

[19] Quigle, RM, Yanful EK, Helganson T, Margaritis A. Hydraulic conductivity of contaminated natural clay directly below a domestic landfill. Can Geotech J 1987; 24: 377-83.

[20] Shakelford CD, Daniel DE. Diffusion in saturated soil ii: results for compacted clay. J Geotech Eng ASCE 1999; 117: 485-506.

(C) Jhamnani and Singh; Licensee Bentham Open.

This is an open access article licensed under the terms of the Creative Commons Attribution Non-Commercial License (http://creativecommons.org/licenses/by-nc/3.0/) which permits unrestricted, non-commercial use, distribution and reproduction in any medium, provided the work is properly cited. 